\title{
Dielectric Permeability of Nanocylinder
}

\author{
S. JaĆImovski ${ }^{a, *}, \mathrm{~V}$. SAJferT ${ }^{b}$, D. RAKOVIĆ ${ }^{c}$ AND B. TOŠIĆ ${ }^{d}$ \\ ${ }^{a}$ Academy of Criminalistic and Police Studies, 196 Cara Dusana St., 11080 Belgrade, Serbia \\ ${ }^{b}$ Technical Faculty "M. Pupin", University of Novi Sad, Zrenjanin, Serbia \\ ${ }^{c}$ Faculty of Electrical Engineering, University of Belgrade, Serbia \\ ${ }^{d}$ Vojvodina Academy of Sciences and Arts, Novi Sad, Serbia
}

\begin{abstract}
In the nanocylinder, a cut-off from the molecular crystal, dielectric permeability tensor is investigated. Excitons in the nanocylinder arise due to the exciting of the electron subsystem of the molecule. In evaluation of dielectric permeability Dzhyaloshinskii-Pitaevskii approach is used, connected with retarded and advanced exciton Green's functions and correct use of Paulion Green's function. It turned out that refraction and absorption indices depend on configuration coordinates, having maximal values at boundary cross-sections and minimal value at central cross-section of the nanocylinder broken symmetry structure. Although it was expected that boundary conditions make higher refractive and absorptive characteristics of the nanocylinder, this appeared not to be possible because Paulion Green's function is not proportional to the exciton concentration.
\end{abstract}

PACS numbers: $78.20 \mathrm{Ci}, 78.40 \mathrm{Me}$

\section{Introduction}

Our goal is to investigate dielectric permeability tensor $\varepsilon_{\alpha \beta}$, as well as tensors of nonlinear polarization of the nanocylinder. In nonlinear optics, the vector of electrical induction $D$ is the series of different degrees of electrical field components. The coefficient in the first term of this series is dielectric permeability tensor, while in other terms appear higher tensors of third range, fourth range etc.

The idea of Dzyalosinski and Pitaevski [1], for equating the phenomenological value of the vector potential from Maxwell's equations with its nonequilibrium average value, is the bridge connecting optical phenomenology with micro characteristic of molecular crystals. The micro characteristic of crystals enters the retarded and advanced Green's functions of excitons [2]. In the case of excitons which arise due to the excitations of electron subsystems, exciton Green's functions are product of Pauli or quasi Pauli operators [3-4]. Pauli operators satisfy the following commutations rules:

$$
\begin{aligned}
& {\left[P_{n}, P_{m}^{+}\right]=\left(1-2 P_{m}^{+} P_{n}\right) \delta_{n, m} ; \quad P_{n}^{2}=\left(P_{n}^{+}\right)^{2}=0 ;} \\
& {\left[P_{n}, P_{m}\right]=\left[P_{n}^{+}, P_{m}^{+}\right]=0 .}
\end{aligned}
$$

The commutation rules for quasi Pauli operators are still more complicated and will be not quoted here.

The problems concerned with the calculation and the use of Paulion Green's function are the following:

1. Fourier transformation of Pauli operators $P_{n}=$ $\frac{1}{\sqrt{N}} \sum_{k} \mathrm{e}^{\mathrm{i} a k n} P_{k}$ is not canonical transformation. It means that operators $P_{k}$ are not Pauli operators.

\footnotetext{
* corresponding author; e-mail: stevo.jacimovski@kpa.edu.rs
}

2. In calculations of average value $\left\langle P_{n}^{+} P_{n}\right\rangle$ by means of spectral intensity of Paulion Green's function, positive correction proportional to square of Paulion concentration appears, well known in literature as spurious error [5].

3. The application of Wick's theorem to Pauli operators' averages is not formulated up to now.

The mentioned arguments degrade Paulion Green's functions as the method in solving of nonlinear problems. This is the reason to start with problem of finding the correct use of Paulion Green's function, which will be done in the next section.

Finally we shall determine Green's functions of the nanocylinder. These Green's functions will be used for determining of dielectric permeability tensor and for finding of nonlinear polarizability tensors of third of fourth range, in order to find their dependence on configuration coordinates in the nanocylinder broken symmetry structure.

\section{Correct expression for Paulion Green's function}

We shall consider here the linear infinite chain of molecules. The procedure derived for linear chain can be then extended for two and three dimensional structures.

The excitonic Hamiltonian of an ideal molecular chain, in the nearest neighbours approximation, can be written as follows:

$$
\begin{aligned}
H_{\mathrm{P}} & =\Delta \sum_{n} P_{n}^{+} P_{n}-W \sum_{n} P_{n}^{+}\left(P_{n+1}+P_{n-1}\right) \\
& -F \sum_{n} P_{n}^{+} P_{n}\left(P_{n+1}^{+}+P_{n+1}+P_{n-1}^{+}+P_{n-1}\right) .
\end{aligned}
$$

Paulion Green's function of the system is defined as: 


$$
\left\langle\left\langle P_{n}(t) \mid P_{m}^{+}(0)\right\rangle\right\rangle=\theta(t)\left\langle\left[P_{n}(t), P_{m}^{+}(0)\right]\right\rangle .
$$

Differentiating Eq. (2.2) with respect to $t$ and using Fourier time-frequency transformation

$$
f(t)=\int_{-\infty}^{+\infty} d \omega \mathrm{e}^{-\mathrm{i} \omega t} f(\omega),
$$

we obtain the following equation for Paulion Green's function:

$$
\begin{aligned}
& (E-\Delta)\left\langle\left\langle P_{n} \mid P_{m}^{+}\right\rangle\right\rangle_{\omega}=\frac{\mathrm{i} \hbar}{2 \pi}\left(1-2 N_{p}\right) \\
& \quad-W\left[\left\langle\left\langle P_{n+1} \mid P_{m}^{+}\right\rangle\right\rangle_{\omega}+\left\langle\left\langle P_{n-1} \mid P_{m}^{+}\right\rangle\right\rangle_{\omega}\right] \\
& +2 W\left(\left\langle\left\langle P_{n}^{+} P_{n} P_{n+1} \mid P_{m}^{+}\right\rangle\right\rangle_{\omega}\right. \\
& +\left\langle\left\langle P_{n}^{+} P_{n} P_{n-1} \mid P_{m}^{+}\right\rangle\right\rangle_{\omega}-2\left\langle\left\langle P_{n+1}^{+} P_{n+1} P_{n} \mid P_{m}^{+}\right\rangle\right\rangle_{\omega} \\
& +\left\langle\left\langle P_{n-1}^{+} P_{n-1} P_{n} \mid P_{m}^{+}\right\rangle\right\rangle_{\omega} ; \quad N_{p} \equiv\left\langle P^{+} P\right\rangle .
\end{aligned}
$$

The higher order Green's function, containing four Pauli operators, will be decoupled by means of Tyablikov approximation $[2,5]$

$$
\begin{aligned}
& \left\langle\left\langle P_{n}^{+}(t) P_{n}(t) P_{l}(t) \mid P_{m}^{+}(0)\right\rangle\right\rangle_{\omega} \approx N_{p 0}=\left\langle\left\langle P_{l} \mid P_{n}^{+}\right\rangle\right\rangle_{\omega} ; \\
& N_{p 0} \equiv\left\langle P^{+} P\right\rangle_{0} .
\end{aligned}
$$

Tyablikov's approximation physically means substitution of the scattering processes on real potential by the propagation of particles through the soft potential.

After inserting Eq. (2.5) into Eq. (2.4) for Paulion Green's function, we have:

$$
\begin{aligned}
& (E-\Delta)\left\langle\left\langle P_{n} \mid P_{m}^{+}\right\rangle\right\rangle_{\omega}=\frac{\mathrm{i} \hbar}{2 \pi} \delta_{n, m}\left(1-2 N_{p}\right) \\
& -\left(1-2 N_{p 0}\right) W\left[\left\langle\left\langle P_{n+1} \mid P_{m}^{+}\right\rangle\right\rangle_{\omega}+\left\langle\left\langle P_{n-1} \mid P_{m}^{+}\right\rangle\right\rangle_{\omega}\right] \\
& \quad-4 F N_{p 0}\left\langle\left\langle P_{n} \mid P_{m}^{+}\right\rangle\right\rangle_{\omega} .
\end{aligned}
$$

Further solving of Eq. (2.6) requires application of non-canonical transformation

$$
P_{n}=\frac{1}{\sqrt{N}} \sum_{n} \mathrm{e}^{\mathrm{i} k n a} P_{k},
$$

which reduces Eq. (2.6) to

$$
\begin{aligned}
& \left\langle\left\langle P \mid P^{+}\right\rangle\right\rangle_{k, \omega}=\frac{\mathrm{i} \hbar}{2 \pi} \frac{1-2 N_{p}}{\mathrm{e}^{-\frac{E_{k}^{(1)}}{\theta}}-1} ; \\
& E_{k}^{(1)}=\Delta-2 W \cos a k+4(W \cos a k-F) N_{p 0} .
\end{aligned}
$$

It should be noticed that correlator of Paulion Green's function (2.8) leads to spurious error [5].

We shall not use Paulion Green's function (2.8) due to the reasons [1-2] given in the Introduction. Instead, we shall use equivalent bozon Green's function, which will be determined by means of the exact bozon representation for Pauli operators from [4]. In the considered case it is sufficient to use this exact representation in the approximations

$$
P=B-B^{+} B B ; \quad P^{+}=B^{+}-B^{+} B^{+} B ;
$$

$$
P^{+} P=B^{+} B-B^{+} B^{+} B B .
$$

Inserting Eq. (2.9) into Eq. (2.6), by means of Wick's theorem for bozons we obtain

$$
\begin{aligned}
(1 & \left.-4 N_{B 0}\right)(E-\Delta)\left\langle\left\langle B_{n} \mid B_{m}^{+}\right\rangle\right\rangle_{\omega} \\
& +\left(1-2 N_{B 0}\right) W\left\langle\left\langle B_{n+1} \mid B_{m}^{+}\right\rangle\right\rangle \\
& +\left(1-2 N_{B 0}\right) W\left\langle\left\langle B_{n-1} \mid B_{m}^{+}\right\rangle\right\rangle \\
& =\frac{\mathrm{i} \hbar}{2 \pi} \delta_{n, m}\left(1-2 N_{B}\right)-4 F N_{B 0}\left\langle\left\langle B_{n} \mid B_{m}^{+}\right\rangle\right\rangle
\end{aligned}
$$

We note that in Eq. (2.10) squares of bozon concentration are neglected.

Using Fourier transformation of Bose operator (which is canonical transformation for bozons), we obtain the following equation for bozon Green's function $\left\langle\left\langle B_{k} \mid B_{k}^{+}\right\rangle\right\rangle_{\omega}$ :

$$
\left\langle\left\langle B_{k} \mid B_{k}^{+}\right\rangle\right\rangle_{\omega}=\frac{\mathrm{i} \hbar}{2 \pi} \frac{1+2 N_{B 0}}{E-E_{k}^{(1)}}
$$

where

$$
E_{k}^{(1)}=\Delta-2 W \cos a k+4(W \cos a k-F) N_{B 0}
$$

The energy of zero approximations is

$$
E_{k}^{(0)}=\Delta-2 W \cos a k
$$

Now we shall calculate zero order value $N_{B 0}$. Taking $\cos a k \approx 1-\frac{1}{2} a^{2} k^{2}+\frac{1}{24} a^{4} k^{4}$, we obtain:

$$
\begin{aligned}
N_{B 0} & =\frac{1}{N} \sum_{k>0} \frac{1}{\mathrm{e}^{\frac{E_{k}^{(0)}}{\theta}}-1} \\
= & \left(\frac{\theta}{4 \pi W}\right)^{1 / 2} \sum_{n=1}^{\infty} \mathrm{e}^{-n\left(\frac{\Delta-2 W}{\theta}\right)} n^{-1 / 2} \\
+ & \frac{\pi}{4}\left(\frac{\theta}{4 \pi W}\right)^{3 / 2} \sum_{n=1}^{\infty} \mathrm{e}^{-n\left(\frac{\Delta-2 W}{\theta}\right)} n^{-3 / 2}
\end{aligned}
$$

For $N_{B}$ we obtain the formula:

$$
\begin{aligned}
& N_{B}=\frac{1}{N} \sum_{k>0} \frac{1}{\mathrm{e}^{\frac{E_{k}^{(1)}}{\theta}}-1}=N_{B 0}-\left\{C_{1 / 2}(\theta)\left(\frac{\theta}{4 \pi W}\right)^{1 / 2}\right. \\
& -C_{3 / 2}(\theta)\left(\frac{\theta}{4 \pi W}\right)^{3 / 2} \\
& \left.+C_{5 / 2}(\theta)\left(\frac{\theta}{4 \pi W}\right)^{5 / 2}\right\} N_{B 0},
\end{aligned}
$$

where

$$
\begin{aligned}
& C_{1 / 2}(\theta)=\frac{4(\mathrm{~W}-\mathrm{F})}{\theta} \sum_{n=1}^{\infty} \mathrm{e}^{-n\left(\frac{\Delta-2 W}{\theta}\right)} n^{1 / 2}, \\
& C_{3 / 2}(\theta)=\pi \frac{(W+F)}{\theta} \sum_{n=1}^{\infty} \mathrm{e}^{-n\left(\frac{\Delta-2 W}{\theta}\right)} n^{-1 / 2}, \\
& C_{5 / 2}(\theta)=\pi^{2} \frac{W}{\theta} \sum_{n=1}^{\infty} \mathrm{e}^{-n\left(\frac{\Delta-2 W}{\theta}\right)} n^{-3 / 2} .
\end{aligned}
$$

Besides, in accordance with Eq. (2.9) we can take 


$$
N_{p}=N_{B}-2 N_{B 0}^{2} .
$$

Multiplying Eq. (2.8) and (2.11) we have

$$
\begin{aligned}
& \left\langle\left\langle P \mid P^{+}\right\rangle\right\rangle_{k \omega}\left\langle\left\langle B \mid B^{+}\right\rangle\right\rangle_{k, \omega} \\
& =\left(\frac{\mathrm{i} \hbar}{2 \pi} \frac{1}{E-E_{k}^{(1)}}\right)^{2}\left(1-2 N_{p}\right)\left(1+2 N_{B 0}\right) \\
& =\left(\frac{\mathrm{i} \hbar}{2 \pi} \frac{1}{E-E_{k}^{(1)}}\right)^{2}\left(1-2\left(N_{B 0}-N_{B}\right)\right. \\
& \left.+4\left(N_{B 0}^{2}-N_{B 0} N_{B}\right)+8 N_{B 0}^{3}\right) .
\end{aligned}
$$

It was said that all contributions proportional to $N_{B 0}^{2}$ and higher degrees of $N_{B 0}^{2}$ are to be neglected in this analysis, so that we can write Eq. (2.20) as

$$
\begin{aligned}
& \left\langle\left\langle P \mid P^{+}\right\rangle\right\rangle_{k \omega}\left\langle\left\langle B \mid B^{+}\right\rangle\right\rangle_{k, \omega} \\
& =\left(\frac{\mathrm{i} \hbar}{2 \pi} \frac{1}{E-E_{k}^{(1)}}\right)^{2}\left(1-2\left(N_{B 0}-N_{B}\right)\right) .
\end{aligned}
$$

Now we shall estimate the value $2\left(N_{B 0}-N_{B}\right)$. Taking only leading terms in $N_{B}$ and $N_{B 0}$ in Eq. (2.10) we have:

$$
\begin{aligned}
& 2\left(N_{B 0}-N_{B}\right)=2 \frac{(\mathrm{W}-\mathrm{F})}{\pi W} \sum_{n=1}^{\infty} \mathrm{e}^{-n\left(\frac{\Delta-2 W}{\theta}\right)} n^{-1 / 2} \\
& \quad \times \sum_{n=1}^{\infty} \mathrm{e}^{-n\left(\frac{\Delta-2 W}{\theta}\right)} n^{-3 / 2} .
\end{aligned}
$$

Since the maximal achieved exciton concentrations are of the order $10^{-3}$ [2] we can take in Eq. (2.22) that $\mathrm{e}^{-\frac{\Delta-2 W}{\theta}}=10^{-3}$. This gives the estimation $2\left(N_{B 0}-\right.$ $\left.N_{B}\right) \approx 10^{-6} \approx N_{B 0}^{2}$. Consequently, the term $2\left(N_{B 0}-\right.$ $N_{B}$ ) in Eq. (2.21) must be neglected and Eq. (2.21) becomes

$$
\left\langle\left\langle P \mid P^{+}\right\rangle\right\rangle_{k \omega}\left\langle\left\langle B \mid B^{+}\right\rangle\right\rangle_{k, \omega}=\left(\frac{\mathrm{i} \hbar}{2 \pi} \frac{1}{E-E_{k}^{(1)}}\right)^{2}
$$

$$
\begin{aligned}
H & =\sum_{n=0}^{N} \sum_{m=0}^{M} \Delta P_{n m}^{+} P_{n m}+\sum_{n=0}^{N} \sum_{m=1}^{M}\left(X_{n, m ; n+1, m}+X_{n, m ; n-1, m}+X_{n, m ; n, m+1}+X_{n, m ; n, m-1}\right) P_{n m}^{+} P_{n m} \\
& -\sum_{n=0}^{N} \sum_{m=1}^{M-1}\left(P_{n, m}^{+} Y_{n, m ; n+1, m} P_{n+1, m}+Y_{n, m ; n-1, m} P_{n-1, m}+Y_{n, m ; n, m+1} P_{n, m+1}+Y_{n, m ; n, m-1} P_{n, m-1}\right) \\
& +\sum_{n=0}^{N} \sum_{m=1}^{M-1} P_{n m}^{+} P_{n m}\left(Z_{n, m ; n+1, m} P_{n+1, m}^{+} P_{n+1, m}\right. \\
& \left.+Z_{n, m ; n-1, m} P_{n-1, m}^{+} P_{n-1, m}+Z_{n, m ; n, m+1} P_{n, m+1}^{+} P_{n, m+1}+Z_{n, m ; n, m-1} P_{n, m-1}^{+} P_{n, m-1}\right) \\
& +\sum_{n=0}^{N} X_{n, 0 ; n, 1} P_{n, 0}^{+} P_{n, 0}-\sum_{n=0}^{N}\left(P_{n, 0}^{+} Y_{n, 0 ; n+1,0} P_{n+1,0}+Y_{n, 0 ; n-1,0} P_{n-1,0}+Y_{n, 0 ; 0,1} P_{n, 1}\right) \\
& +\sum_{n=0}^{N} P_{n 0}^{+} P_{n 0}\left(Z_{n, 0 ; n+1,0} P_{n+1,0}^{+} P_{n+1,0}+Z_{n, 0 ; n-1,0} P_{n-1,0}^{+} P_{n-1,0}+Z_{n, 0 ; n, 1} P_{n, 1}^{+} P_{n, 1}\right)
\end{aligned}
$$

It should be pointed out that this correct use of Paulion Green's functions does not lead to spuriours error, and that gives the correct expression for low temperature magnetization in the theory of magnetism.

\section{Green's functions of nanocylinder}

Nanocylinder will be taken as a set of parallel discs normal to $z$ axis. Number of $\operatorname{discs} M$ is of the order 10. Number $N$ of molecules in disc is also maximally of the order 10 . The discs will be labeled with integer $m \in(0, M)$. The molecules in disc will be labeled with $n \in(0, N)[6]$

boundary conditions are actual along $z$ axis. In $M+1$ are absent. So the boundary conditions can be ritten as:

$$
\begin{aligned}
& X_{n, 0 ; n,-1}=X_{n, M ; n, M+1} ; \quad Y_{n, 0 ; n,-1}=Y_{n, M ; n, M+1} ; \\
& Z_{n, 0 ; n,-1}=Z_{n, M ; n, M+1} .
\end{aligned}
$$
neighbour molecules in one disc and interaction between corresponding molecules laying in neighbour discs differ, the Hamiltonian of nanocylinder we can written in the nearest neighbours approximation as follows:

ne of molecules is excited by energy 


$$
\begin{aligned}
& +\sum_{n=0}^{N} X_{n, M ; n, M-1} P_{n, M}^{+} P_{n, M}-\sum_{n=0}^{N}\left(P_{n, M}^{+} Y_{n, M ; n+1, M} P_{n+1, M}+Y_{n, M ; n-1, M} P_{n-1, M}+Y_{n, M ; n, M-1} P_{n, M-1}\right) \\
& +\sum_{n=0}^{N} P_{n M}^{+} P_{n M}\left(Z_{n, M ; n+1, M} P_{n+1, M}^{+} P_{n+1, M}+Z_{n, M ; n-1, M} P_{n-1, M}^{+} P_{n-1, M}+Z_{n, M ; n, M-1} P_{n, M-1}^{+} P_{n, M-1}\right) .
\end{aligned}
$$

In this Hamiltonian $\Delta \approx 5 \mathrm{eV}$ is the energy of excitation of an isolated molecule [7-9], while $X, Y$, and $Z$ are matrix elements of the dipole-dipole interaction which are two orders of magnitude less than $\Delta$. The terms proportional to $Z$ characterize dynamical interaction of excitons.

It is important to note that system of molecules in disc is cyclically invariant. It means that for arbitrary physical characteristic $F$ the following is valid:

$$
F_{n}=F_{n+(N+1)} \text {. }
$$

For further evaluation it is important to point out the cyclic rules $P_{n+(N+1)}=P_{n}$ and $\mathrm{e}^{\mathrm{i} a k n}=\mathrm{e}^{\mathrm{i} a k(n+(N+1))}$, wherefrom $\mathrm{e}^{\mathrm{i} a k(n+1)}=1$. The last means that $k=\frac{2 \pi \nu}{N+1}$, where $\nu$ is an integer. On this basis, Kronecker symbol can be expressed in terms of plane waves:

$$
\begin{aligned}
\delta_{k, q} & =\frac{1}{N+1} \sum_{n=0}^{N} \mathrm{e}^{\mathrm{i} a(k-q) n} \\
& =\left\{\begin{array}{cc}
1 & \text { if } k=q \\
\frac{1-\mathrm{e}^{\mathrm{i} a(k-q)(n+1)}}{1-\mathrm{e}^{\mathrm{i} a(k-q)}}=0 & \text { if } k \neq q
\end{array}\right.
\end{aligned}
$$

allowing that the system of disc molecules can be treated as an ideal structure.

Introducing notations

$$
\begin{aligned}
& X_{n, m ; n \pm 1, m}=C ; \quad Y_{n, m ; n \pm 1, m}=-D ; \\
& X_{n, m ; n, m \pm 1}=R ; \quad Y_{n, m ; n, m \pm 1}=-S ; \\
& Z_{n, m ; n \pm 1, m}=-F ; \quad Z_{n, m ; n, m \pm 1}=-L,
\end{aligned}
$$

$$
\begin{aligned}
H & =\sum_{n=0}^{N} \sum_{m=1}^{M} \Delta P_{n, m}^{+} P_{n, m} \\
& +\sum_{n=0}^{N} \sum_{m=1}^{M-1}\left[2(C+R) P_{n, m}^{+} P_{n, m}-D\left(P_{n, m}^{+} P_{n+1, m}+P_{n, m}^{+} P_{n-1, m}\right)-S\left(P_{n, m}^{+} P_{n, m+1}+P_{n, m}^{+} P_{n, m-1}\right)\right] \\
& -\sum_{n=0}^{N} \sum_{m=1}^{M-1}\left[F P_{n, m}^{+} P_{n, m}\left(P_{n+1, m}^{+} P_{n+1, m}+P_{n-1, m}^{+} P_{n-1, m}\right)+L P_{n, m}^{+} P_{n, m}\left(P_{n, m+1}^{+} P_{n, m+1}+P_{n, m-1}^{+} P_{n, m-1}\right)\right] \\
& +C \sum_{n=0}^{N} P_{n, 0}^{+} P_{n, 0}-\sum_{n=0}^{N}\left(P_{n, 0}^{+} D P_{n+1,0}+D P_{n-1,0}+R P_{n, 1}\right) \\
& +\sum_{n=0}^{N} P_{n, 0}^{+} P_{n, 0}\left(F P_{n+1,0}^{+} P_{n+1,0}+F P_{n-1,0}^{+} P_{n-1,0}+L P_{n, 1}^{+} P_{n, 1}\right) \\
& +C \sum_{n=0}^{N} P_{n, M}^{+} P_{n, M}-\sum_{n=0}^{N}\left(P_{n, M}^{+} D P_{n+1, M}+D P_{n-1, M}+R P_{n, M-1}\right) \\
& +\sum_{n=0}^{N} P_{n, M}^{+} P_{n, M}\left(F P_{n+1, M}^{+} P_{n+1, M}+F P_{n-1, M}^{+} P_{n-1, M}+L P_{n, M-1}^{+} P_{n, M-1}\right)
\end{aligned}
$$

Paulion Green's function will be denoted as:

$$
\begin{aligned}
& \Gamma_{n, m ; n^{\prime}, m^{\prime}}\left(t, t^{\prime}\right)=\theta\left(t-t^{\prime}\right)\left\langle\left[P_{n, m}(t), P_{n^{\prime}, m^{\prime}}^{+}\right]\right\rangle \\
& \quad \equiv\left\langle\left\langle P_{n, m}(t) \mid P_{n^{\prime}, m^{\prime}}^{+}\right\rangle\right\rangle .
\end{aligned}
$$

It will be determined by standard Green's function solving procedure, although all problems pointed out in introduction stay opened.
Differentiating Eq. (3.7) with respect to $t$, taking into account equations of motion, and using transformation

$$
\begin{aligned}
& \Gamma_{n, m ; n^{\prime} m^{\prime}}(\omega) \\
& \quad=\frac{1}{N+1} \sum_{\nu} \mathrm{e}^{\mathrm{i}\left(n-n^{\prime}\right) \tau_{\nu}} \gamma_{m, m^{\prime}}(\omega, \nu),
\end{aligned}
$$

with 


$$
\tau_{\nu}=\frac{2 \pi \nu}{N+1},
$$

we obtain the following system of three difference equation [10]:

$$
\begin{aligned}
& \tilde{S}\left(\gamma_{m+1, m}+\gamma_{m-1, m}\right)+\rho \gamma_{m, m^{\prime}} \\
& \quad=\frac{\mathrm{i} \hbar}{2 \pi}\left(1-2 N_{\mathrm{P}}\right) \delta_{m, m^{\prime}} ; \quad 1 \leq m \leq M-1 \\
& \tilde{S} \gamma_{1, m^{\prime}}+\left(\rho+R-L N_{\mathrm{P} 0}\right) \gamma_{0, m^{\prime}} \\
& \quad=\frac{\mathrm{i} \hbar}{2 \pi}\left(1-2 N_{\mathrm{P}}\right) \delta_{m, m^{\prime}} ; \quad m=0 \\
& \tilde{S} \gamma_{\mathrm{M}-1, \mathrm{~m}^{\prime}}+\left(\rho+R-L N_{\mathrm{P} 0}\right) \gamma_{\mathrm{M}, \mathrm{m}^{\prime}} \\
& \quad=\frac{\mathrm{i} \hbar}{2 \pi}\left(1-2 N_{\mathrm{P}}\right) \delta_{\mathrm{M}, \mathrm{m}^{\prime}} ; \quad m=M,
\end{aligned}
$$

where

$$
\begin{aligned}
& \tilde{S}=S\left(1-2 N_{\mathrm{P}}\right) ; \quad \tilde{D}=D\left(1-2 N_{\mathrm{P}}\right) ; \\
& \rho=E-\Delta-2 D-2 R+2 \tilde{D} \cos \tau_{\nu} ; \\
& \left\langle B_{n, m}^{+} B_{n, m}\right\rangle^{(0)}=N_{B 0} \approx\left\langle P_{n, m}^{+} P_{n, m}\right\rangle^{(0)}=N_{\mathrm{P} 0} ; \\
& \left\langle B_{n, m}^{+} B_{n, m}\right\rangle=N_{B} .
\end{aligned}
$$

By the substitution

$$
\gamma_{m, m^{\prime}}(\omega, \nu)=\sum_{\sigma} A_{\sigma}\left(m^{\prime}, \nu, \omega\right) \Lambda(m),
$$

where

$$
\Lambda(m)=\sin (m+1) \varphi_{\sigma}-\frac{R-L N_{\mathrm{P} 0}}{S\left(1-2 N_{\mathrm{P} 0}\right)} \sin m \varphi_{\sigma},
$$

and parameter $\varphi_{\sigma}$ satisfy the equation:

$$
\begin{aligned}
& \sin (M+2) \varphi_{\sigma}-2 \frac{R-L N_{\mathrm{P} 0}}{S\left(1-N_{\mathrm{P} 0}\right)} \sin (M+1) \varphi_{\sigma} \\
& \quad+\left[\frac{R-L N_{\mathrm{P} 0}}{S\left(1-2 N_{\mathrm{P} 0}\right)}\right]^{2} \sin M \varphi_{\sigma}=0,
\end{aligned}
$$

the system of Eq. (3.10-3.12) reduces into one unique equation:

$$
\begin{aligned}
& \sum_{\sigma}\left(2 \tilde{S} \cos \varphi_{\sigma}+\rho\right) A_{\sigma}\left(m^{\prime}, \omega, \nu\right) \Psi_{\sigma}(m) \\
& \quad=\frac{\mathrm{i} \hbar}{2 \pi}\left(1+2 N_{B 0} \delta_{m, m^{\prime}}\right), \quad m, m^{\prime} \in(0,1,2, \ldots, N) .
\end{aligned}
$$

Parameter $\varphi_{\sigma}$ have real solutions of the Eq. (3.16), laying in the interval $(0, \pi)$. Parameter $\varphi_{\sigma}$ may not have values $m=0$ or $m=M$, since in these cases the Green's function becomes $\gamma \equiv 0$. Also, in the system the autoreduction process takes place. It means, that $m$ takes $m+1$ values for $m$ values of $\sigma$. It means that we must in principle solve two problems: one problem is related to the subsystem of $m \in(0,30)$ and the second problem is related to the subsystem $m \in(1,31)$.

Taking that

$$
A_{\sigma}\left(m^{\prime}, \nu, \omega\right)=\chi(\omega, \nu) \Phi_{\sigma}\left(m^{\prime}\right),
$$

and

$$
\sum_{\sigma} \Lambda_{\sigma}(\omega, \nu) \Phi\left(m^{\prime}\right)=\delta_{n, m},
$$

since $\chi_{\sigma}(\omega) \equiv\left\langle\left\langle P^{+} \mid P\right\rangle\right\rangle$, we can finally write the expression for Fourier image of the Paulion Green's function:

$$
\left\langle\left\langle P \mid P^{+}\right\rangle\right\rangle_{\sigma, \omega}=\frac{\mathrm{i} \hbar}{2 \pi} \frac{1-2 N_{\mathrm{P}}}{E-E_{\sigma}^{(1)}},
$$

where

$$
\begin{gathered}
E_{\sigma}^{(1)}=\Delta+2 C+2 R-2 D\left(1-2 N_{B 0}\right) \cos \tau_{\nu} \\
-2 S\left(1-2 N_{B 0}\right) \cos \varphi_{\sigma}-4(F+L) N_{B 0} .
\end{gathered}
$$

Now we shall look for bozon Green's function of the nanocylinder. This bozon Green's function is given by:

$$
\begin{aligned}
& G_{n, m ; n^{\prime}, m^{\prime}}\left(t, t^{\prime}\right)=\theta\left(t-t^{\prime}\right)\left\langle\left[B_{n, m}(t), B_{n^{\prime}, m^{\prime}}^{+}\right]\right\rangle \\
& \quad \equiv\left\langle\left\langle B_{n, m}(t) \mid B_{n^{\prime}, m^{\prime}}^{+}\right\rangle\right\rangle .
\end{aligned}
$$

By inserting Eq. (2.9) into Eq. (3.7), by absolutely same procedure we can determine the bozon Green's function:

$$
\left\langle\left\langle B \mid B^{+}\right\rangle\right\rangle_{\sigma, \omega}=\frac{\mathrm{i} \hbar}{2 \pi} \frac{1+2 N_{B 0}}{E-E_{\sigma}^{(1)}} .
$$

Multiplying Eq. (3.23) and (3.20) we obtain:

$$
\begin{aligned}
& \left\langle\left\langle P \mid P^{+}\right\rangle\right\rangle_{k \omega}\left\langle\left\langle B \mid B^{+}\right\rangle\right\rangle_{k \omega} \\
& =\left(\frac{\mathrm{i} \hbar}{2 \pi} \frac{1}{E-E_{k}^{(1)}}\right)^{2}\left(1-2 N_{p}\right)\left(1+2 N_{B 0}\right) \\
& =\left(\frac{\mathrm{i} \hbar}{2 \pi} \frac{1}{E-E_{k}^{(1)}}\right)^{2}\left(1-2\left(N_{B 0}-N_{B}\right)\right. \\
& \left.+4\left(N_{B 0}^{2}-N_{B 0} N_{B}\right)+8 N_{B 0}^{3}\right) .
\end{aligned}
$$

In the first section it was demonstrated that all terms added to unit on the right hand side of the Eq. (3.24) are of the order $N_{B 0}^{2}$, i.e. of the order $10^{-6}$. This is the reason to neglect all these terms. It means that $\left\langle\left\langle P \mid P^{+}\right\rangle\right\rangle_{\sigma, \omega}\left\langle\left\langle B \mid B^{+}\right\rangle\right\rangle_{\sigma, \omega}$ reduces to

$$
\left\langle\left\langle P \mid P^{+}\right\rangle\right\rangle_{\sigma, \omega}\left\langle\left\langle B \mid B^{+}\right\rangle\right\rangle_{\sigma, \omega}=\left(\frac{\mathrm{i} \hbar}{2 \pi} \frac{1}{E-E_{\sigma}^{(1)}}\right)^{2} .
$$

It means that Paulion Green's function, which enters dielectric permeability and nonlinear polarizability tensor, is given by the Eq. (3.25).

\section{Dielectric polarization of crystal}

The dielectric permeability tensor $\varepsilon_{\alpha \beta}(\boldsymbol{k}, \omega)$ represents the relation between vacuum electrical field and its images in the material medium induction vector $\boldsymbol{D}$. In Fourier components this relation is given by the equation

$$
\boldsymbol{D}_{i}(\boldsymbol{k}, \omega)=\varepsilon_{\alpha \beta}(\boldsymbol{k}, \omega) E_{j}(\boldsymbol{k}, \omega) .
$$

In accordance with Einstein convention, repeated index means summation. The system of Maxwell's equations, where weak external currents are present and external charges are absent, can be written as follows: 


$$
\begin{aligned}
\boldsymbol{k} \times \boldsymbol{B}(\boldsymbol{k}, \omega) & =-\frac{\omega}{c} \boldsymbol{D}(\boldsymbol{k}, \omega)+\frac{4 \pi}{\mathrm{i} c} j^{\mathrm{ext}}(\boldsymbol{k}, \omega), \\
\boldsymbol{k} \times \boldsymbol{D}(\boldsymbol{k}, \omega) & =0 \\
\boldsymbol{k} \times \boldsymbol{E}(\boldsymbol{k}, \omega) & =\frac{\omega}{c} \boldsymbol{B}(\boldsymbol{k}, \omega), \\
\boldsymbol{k} \times \boldsymbol{B}(\boldsymbol{k}, \omega) & =0 .
\end{aligned}
$$

Since external charges are equal to zero the following conditions are valid:

$$
\boldsymbol{k} \cdot \boldsymbol{D}(\boldsymbol{k}, \omega)=\boldsymbol{k} \cdot \boldsymbol{B}(\boldsymbol{k}, \omega)=0
$$

If we use Lorentz calibration of vector potential $\boldsymbol{A}(\boldsymbol{k}, \omega)$, we obtain equations:

$$
\begin{aligned}
& \boldsymbol{B}(\boldsymbol{k}, \omega)=\boldsymbol{r o t} \boldsymbol{A}(\boldsymbol{k}, \omega), \\
& \boldsymbol{E}(\boldsymbol{k}, \omega)=-\frac{1}{c} \frac{\partial \boldsymbol{A}(\boldsymbol{k}, \omega)}{\partial t} .
\end{aligned}
$$

By combining Maxwell's Eq. (4.2), (4.4), and (4.1) with Eq. (4.7) we obtain

$$
\boldsymbol{E}(\boldsymbol{k}, \omega)=\frac{\mathrm{i} \omega}{c} \boldsymbol{A}(\boldsymbol{k}, \omega)
$$

Then we obtain the following relation between the vector potential and the external forces:

$$
\Delta_{\alpha \beta}(\boldsymbol{k}, \omega) A_{\beta}(\boldsymbol{k}, \omega)=\frac{4 \pi}{c} j^{\text {ext }}(\boldsymbol{k}, \omega) .
$$

Consequently, the explicit relation for the vector potential components is [2]:

$$
\Delta_{\alpha \beta}(\boldsymbol{k}, \omega)=k^{2} \delta_{\alpha \beta}-k_{\alpha} k_{\beta}-\frac{\omega^{2}}{c^{2}} \varepsilon_{\alpha \beta}(\boldsymbol{k}, \omega),
$$

and

$$
A_{\beta}(\boldsymbol{k}, \omega)=\frac{4 \pi}{c} \Delta_{\alpha \beta}^{-1}(\boldsymbol{k}, \omega) j^{\mathrm{ext}}(\boldsymbol{k}, \omega) .
$$

This phenomenological value will be equated with the non-equilibrium mean value vector potential. The interaction of the vector potential with the external currents is defined as follows. The non-equilibrium mean value vector potential we shall calculate by using $\mathrm{S}$ matrix of the system, i.e.:

$$
\begin{gathered}
\left\langle A_{\beta}(\boldsymbol{r}, t)\right\rangle_{n}=\left\langle T \mathrm{e}^{-\frac{1}{\mathrm{i} \hbar} \int_{-\infty}^{+\infty} \mathrm{d} t^{\prime} W\left(t^{\prime}\right)}\right. \\
\left.\quad \times A_{\alpha}(\boldsymbol{r}, t) T \mathrm{e}^{\frac{1}{\mathrm{i} \hbar} \int_{-\infty}^{+\infty} \mathrm{d} t^{\prime} W\left(t^{\prime}\right)}\right\rangle .
\end{gathered}
$$

In linear approximation in $j^{\text {ext }}$, we obtain non-equilibrium value of vector potential.

Vector potential has not physical sense. It is only useful mathematical element of the theory. That is the reason for going over of vector potential components to components of electrical field, since electrical field components are measurable. The final connecting of phenomenological electromagnetic field and micro characteristic of physical medium is given by formula [11-12]:

$$
\begin{aligned}
& \Delta_{\alpha \beta}^{-1}(\boldsymbol{k}, \omega)=-\frac{c^{2}}{\omega^{2}} \delta_{\alpha \beta}+\mathrm{i} \frac{c^{2}}{\omega^{2}} \frac{a^{\mathrm{e}} E_{\alpha}(\boldsymbol{k}) E_{\beta}(\boldsymbol{k})}{4 \hbar} \\
& \quad \times\left[G^{R}(\boldsymbol{k}, \omega)+G^{A}(\boldsymbol{k}, \omega)\right],
\end{aligned}
$$

where $\Delta_{\alpha \beta}(\boldsymbol{k}, \omega)$ is given by Eq. (4.10).

The obtained relation is complicated for analysis, so in practice it is used in homogenous approximation and with neglecting of space dispersion [12]. Then general Eq. (4.13) can be written in the form:

$$
\begin{aligned}
& \varepsilon_{\alpha \beta}^{-1}(\boldsymbol{k}, \omega) \\
& \quad=1+\frac{a^{3} E(\boldsymbol{k})}{8 \pi \hbar \omega}\left[\frac{1}{1-\frac{\omega(\boldsymbol{k})}{\omega}}-\frac{1}{1+\frac{\omega(\boldsymbol{k})}{\omega}}\right] .
\end{aligned}
$$

Retarded Green's function $G^{R}(\nu, \omega)$ is given by formula

$$
G^{R}(\nu, \omega)=\frac{\mathrm{i}}{2 \pi} \sum_{\sigma=1}^{M} \frac{\Psi_{\sigma}(m) \Phi_{\sigma}\left(m^{\prime}\right)}{\omega-\omega_{1}} .
$$

Advanced Green's function $G^{A}(\nu, \omega)$ can be obtain from Eq. (4.15) by inversion $\omega \rightarrow-\omega$. Consequently

$$
G^{A}(\nu, \omega)=\frac{\mathrm{i}}{2 \pi} \sum_{\sigma=1}^{M} \frac{\Psi_{\sigma}(m) \Phi_{\sigma}\left(m^{\prime}\right)}{\omega+\omega_{1}} .
$$

Putting Eq. (4.15) and (4.16) into Eq. (4.14) we obtain

$$
\begin{aligned}
& \varepsilon_{\alpha \beta}^{-1}(\boldsymbol{k}, \omega)=1+\frac{a^{3} E^{2}(\boldsymbol{k})}{8 \pi \hbar \omega}\left[\sum_{\sigma=1}^{M} \Psi_{\sigma}(m) \Phi_{\sigma}\left(m^{\prime}\right)\right. \\
& \left.\quad \times\left(\frac{1}{1-\frac{\omega(\boldsymbol{k})}{\omega}}-\frac{1}{1+\frac{\omega(\boldsymbol{k})}{\omega}}\right)\right] .
\end{aligned}
$$

The obtained result points out that dielectric permeability $\varepsilon$ of the nanocylinder depends on the configuration variables $m$ i $m^{\prime}$, and this is the main difference compared to permeability of the ideal structure. With suitable choice of the functions $\Psi$ and $\Phi$, we can influence the magnitude of permeability.

Ending these analyzes, we should say that after construction of the high-power lasers it was registered that material equation is not linear relation between vectors $D$ and $E$. Vector $D$ is infinite series containing products of electrical fields components. The coefficient of this series is called tensor of nonlinear polarizability. Relation $D_{\alpha}=\varepsilon_{\alpha \beta} E_{\beta}$ is only first term of the series [12]

$$
D_{\alpha}=\varepsilon_{\alpha \beta} E_{\beta}+\varepsilon_{\alpha \beta \gamma} E_{\alpha} E_{\beta \gamma}+\varepsilon_{\alpha \beta \gamma \delta} E_{\alpha} E_{\beta \gamma \delta}+\ldots
$$

The tensors $\varepsilon_{\alpha \beta \gamma}$ and $\varepsilon_{\alpha \beta \gamma \delta}$ are so called nonlinear polarizability tensors. In the last formula, as well as in all formulas of this section, repeated index denotes a summation.

\section{Conclusion}

Nanostructures are physical objects of great potential practical interest. Here we investigated what could be expected from nanocylinders in the linear and nonlinear optics.

Dielectric properties of nanocylinder of finite length were therefore analyzed. Defining the correct value of Paulion Green's functions, we determined dielectric permeability of the nanocylinder in $z$-direction, and found that it depends on configuration coordinates. The expressions for transformation functions $\Psi$ and $\Phi$ give possibility to change behaviour of the nanocylinder polarizability. 
It was expected that boundary conditions make higher refractive and absorptive characteristics of the nanocylinder, but this was not possible because Paulion Green's function is not proportional to the exciton concentration. Although negative, this conclusion represents the main result of our analysis.

\section{Acknowledgments}

This paper was in part supported by the Serbian Ministry of Science and Technology: Grant No 141044 and by Vojvodina Academy of Sciences and Arts and by the Provincial Secretariat for Science and Technological Development of the Autonomous Province of Vojvodina (114-451-01336/2007-02).

\section{References}

[1] I.E. Dzyalosinskii, L.P. Piatevskii, ZhETF 36, 1977 (1959).

[2] V.M. Agranovich, Theory of Excitons, 352-356, Nauka, Moscow 1978.
[3] D.I. Lalović, B.S. Tošić, R.B. Žakula, Phys. Rev. 178, 1472 (1969).

[4] V.M. Agranovich, B.S. Tošić, ZhETPh 53, 149 (1967).

[5] S.V. Tyablikov, Methods of Quantum Theory in Magnetism, 232-236 Nauka, Moscow 1975.

[6] M. Laroche, Phys. Rev. B 74, 245422 (2006).

[7] M. Knupfer, T. Schwieger, J. Fink, K. Leo, M. Hoffman, Phys. Rev. B 66, 035208 (2002)

[8] I. Vragović, R. Scholz, M. Schreiber, Europhys. Lett. 57, 288 (2002).

[9] S.E. Sburlan, Phys. Rev. B 73, 035403 (2006).

[10] B. Tošić, V. Sajfert, D. Popov, J. Šetrajčić, D. Ćirić, Application of Difference Calculation in Analyses of Nanostructures, VANU, 131-156 Novi Sad 2005.

[11] V.M. Agranovich, Y.R. Shen, R.H. Baughman, A.A. Zakhidov, Phys. Rev. B 69, 165112 (2004).

[12] U. Kozmidis-Luburić, B. Tošić, Optical Excitations in Material Media, University of Novi Sad, 64-76 Novi Sad 2000. 